\title{
3-Methylxanthine production through biodegradation of theobromine by Aspergillus sydowii PT-2
}

\author{
Binxing Zhou ${ }^{1 *}$ D, Cunqiang Ma ${ }^{1,2,3^{*}+}$, Chengqin Zheng ${ }^{1}$, Tao Xia ${ }^{4}$, Bingsong Ma ${ }^{1}$ and Xiaohui Liu ${ }^{1}$
}

\begin{abstract}
Background: Methylxanthines, including caffeine, theobromine and theophylline, are natural and synthetic compounds in tea, which could be metabolized by certain kinds of bacteria and fungi. Previous studies confirmed that several microbial isolates from Pu-erh tea could degrade and convert caffeine and theophylline. We speculated that these candidate isolates also could degrade and convert theobromine through $\mathrm{N}$-demethylation and oxidation. In this study, seven tea-derived fungal strains were inoculated into various theobromine agar medias and theobromine liquid mediums to assess their capacity in theobromine utilization. Related metabolites with theobromine degradation were detected by using HPLC in the liquid culture to investigate their potential application in the production of 3-methylxanthine.

Results: Based on theobromine utilization capacity, Aspergillus niger PT-1, Aspergillus sydowii PT-2, Aspergillus ustus PT-6 and Aspergillus tamarii PT-7 have demonstrated the potential for theobromine biodegradation. Particularly, $A$. sydowii PT-2 and A. tamarii PT-7 could degrade theobromine significantly $(p<0.05)$ in all given liquid mediums. 3,7Dimethyluric acid, 3-methylxanthine, 7-methylxanthine, 3-methyluric acid, xanthine, and uric acid were detected in A. sydowii PT-2 and A. tamarii PT-7 culture, respectively, which confirmed the existence of N-demethylation and oxidation in theobromine catabolism. 3-Methylxanthine was common and main demethylated metabolite of theobromine in the liquid culture. 3-Methylxanthine in A. sydowii PT-2 culture showed a linear relation with initial theobromine concentrations that $177.12 \pm 14.06 \mathrm{mg} / \mathrm{L}$ 3-methylxanthine was accumulated in TLM-S with $300 \mathrm{mg} / \mathrm{L}$ theobromine. Additionally, $\mathrm{pH}$ at 5 and metal ion of $\mathrm{Fe}^{2+}$ promoted 3-methylxanthine production significantly $(p<$ 0.05).

Conclusions: This study is the first to confirm that A. sydowii PT-2 and A. tamarii PT-7 degrade theobromine through $\mathrm{N}$-demethylation and oxidation, respectively. A. sydowii PT-2 showed the potential application in 3methylxanthine production with theobromine as feedstock through the N-demethylation at N-7 position.
\end{abstract}

Keywords: Aspergillus, Tea, Bioconversion, Theobromine, 3-Methylxanthine

\footnotetext{
*Correspondence: bxzhou01@126.com; macunqiang1208@aliyun.com

${ }^{\dagger}$ Binxing Zhou and Cunqiang Ma contributed equally to this work.

${ }^{1}$ College of Longrun Pu-erh Tea, Yunnan Agricultural University, Kunming 650201, Yunnan, China

Full list of author information is available at the end of the article
}

(C) The Author(s). 2020 Open Access This article is licensed under a Creative Commons Attribution 4.0 International License, which permits use, sharing, adaptation, distribution and reproduction in any medium or format, as long as you give appropriate credit to the original author(s) and the source, provide a link to the Creative Commons licence, and indicate if changes were made. The images or other third party material in this article are included in the article's Creative Commons licence, unless indicated otherwise in a credit line to the material. If material is not included in the article's Creative Commons licence and your intended use is not permitted by statutory regulation or exceeds the permitted use, you will need to obtain permission directly from the copyright holder. To view a copy of this licence, visit http://creativecommons.org/licenses/by/4.0/. The Creative Commons Public Domain Dedication waiver (http://creativecommons.org/publicdomain/zero/1.0/) applies to the data made available in this article, unless otherwise stated in a credit line to the data. 


\section{Background}

Methylxanthines are natural and synthetic compounds found in many foods, drinks, pharmaceuticals, and cosmetics [1]. Caffeine (1,3,7-trimethylxanthine), theobromine (3,7-dimethylxanthine) and theophylline (1,3dimethylxanthine) are most popular and well-known methylxanthines in tea [2]. Both theobromine and theophylline have a close connection with caffeine metabolism in the physiology of tea plant (Camellia sinensis), and the former is precursor of caffeine biosynthesis and the latter is a transient metabolite of caffeine biodegradation [3, 4]. Caffeine level remains stable in the processing of general teas (green tea, black tea, oolong tea and white tea) $[5,6]$. However, the participation of various microorganisms induced the change of caffeine content in the processing of Pu-erh tea and other dark teas $[7,8]$.

$\mathrm{Pu}$-erh tea is a Chinese dark tea produced mainly in Yunan province [9]. Its unique taste and aroma is achieved by natural microorganisms involved in solidstate fermentation [10]. Microorganisms, including bacteria and fungi, have profound impact on substance metabolisms and contributed to the quality formation of $\mathrm{Pu}$-erh tea [11-13]. Aspergillus niger, Aspergillus tubingensis, Aspergillus fumigatus, Aspergillus luchuensis, Aspergillus awamori, Aspergillus tamarii, Blastobotrys adeninivorans, Candida tropicalis, Fusarium graminearum, Pichia farinosa, Rasamsonia byssochlamydoides, Rasamsonia emersonii, Rasamsonia cylindrospora, Rhizomucor pusillus, Rhizomucor tauricus and Thermomyces lanuginosus were detected consecutively in $\mathrm{Pu}$-erh tea [14-17]. Among seven microbial isolates from Pu-erh tea, Aspergillus sydowii have been confirmed to convert degraded caffeine to theophylline, Aspergillus ustus and A. tamarii showed theophylline degradation capacity in liquid culture, respectively [18-20].

Methylxanthines are extensively metabolized in the liver by the cytochrome P450 (CYP450) oxidase enzyme system, mainly through related $\mathrm{N}$-demethylation and oxidation [21]. Although caffeine and other methylxanthines are toxic to most bacteria and invertebrates [22], several bacteria and fungi, including Pseudomonas sp. [23, 24], Pseudomonas putida [25, 26], Serratia marcescens, Fusarium solani [27, 28], Stemphyllium sp., A. tamarii and Penicillium commune [29], have evolved the ability to metabolize caffeine. Two possible pathways of caffeine catabolism, such as $\mathrm{N}$-demethylation and oxidation, are found in microorganisms, which are similar to that in animals and humans [30]. More than one Ndemethylases and oxidases, such as caffeine oxidase, xanthine oxidase and theobromine oxidase, participate into the N-demethylation and oxidation [31-33]. Genes ( $n d m A, n d m B, n d m C, n d m D$ and $n d m E$ ) isolated from $P$. putida are responsible for the entire demethylation pathway [34, 35]. Additionally, genes $c d h A B C$ and
tmuDHM identified in Pseudomonas sp. strain CBB1 are associated with the oxidation of caffeine and trimethyluric acid, respectively $[36,37]$. Therefore, we speculated that those seven microbial isolates from $\mathrm{Pu}$-erh tea also could degrade and convert theobromine through $\mathrm{N}$ demethylation and oxidation.

As the second most common methylxanthine in tea, theobromine dilates blood vessels, especially coronary arteries, lowers blood pressure and increases heart rate [38]. Until now, a bacterial strain $P$. putida isolated from tea garden soil was demonstrated to degrade theobromine [26]. Additionally, A. niger, Talaromyces marneffei and Talaromyces verruculosus isolated from cocoa pod husks were demonstrated to degrade theobromine [39]. In this work, seven tea-derived fungal strains isolated from Pu-erh tea were used to investigate their capacity and characterization in theobromine degradation. It is confirmed that Aspergillus sydowii PT-2 and Aspergillus tamarii PT-7 could degrade theobromine in the liquid culture. Analysis of theobromine degradation metabolites and pathways revealed that 3-methylxanthine was main degradation product of theobromine in A. sydowii PT-2 culture through the $\mathrm{N}$-demethylation at $\mathrm{N}-7$ position. The results showed the application of A. sydowii PT-2 in the production of 3-methylxanthine with theobromine as feedstock.

\section{Results}

Evaluation results of tea-derived fungi in theobromine utilization

To assess theobromine utilization capacity of tea-derived fungi, each microbial isolate was inoculated into different theobromine agar medias (TAM) and theobromine liquid mediums (TLM), respectively. Colony diameters and theobromine concentrations were determined after cultivation at $30^{\circ} \mathrm{C}$ for 5 days. Colony diameters and sporulation time on TAM are recorded in Table 1, and theobromine concentrations in TLM are presented in Fig. 1. As shown in Table 1, apart from Aspergillus pallidofulvus PT-3 and Penicillium mangini PT-5, other microbial isolates had relatively high theobromine utilization capacity, such as Aspergillus niger PT-1, A. sydowii PT-2, Aspergillus sesamicola PT-4, Aspergillus ustus PT-6 and A. tamarii PT-7. Comparison of colony diameters on different TAM showed that dextrose or sucrose as carbon source could promote theobromine utilization partly. TAM-S with the maximal colony diameter was most suitable for theobromine utilization by candidate fungal strains.

In this study, TLM-S, TLM-D, TLM-N, TLM-SN were prepared to select potential theobromine-degrading fungi and optimal medium in the liquid culture. As shown in Fig. 1, due to the difference in cultivation modes, A. pallidofulvus PT-3, A. sesamicola PT-4 and $P$. mangini PT-5 could not utilize theobromine completely 
Table 1 Colony diameter and sporulation time of tea-derived fungi on theobromine agar medias

\begin{tabular}{|c|c|c|c|c|c|c|c|c|c|}
\hline \multirow[t]{2}{*}{ Tea-derived fungi } & \multicolumn{4}{|c|}{ Colony diameter (cm) } & \multirow{2}{*}{$\begin{array}{l}\text { Total } \\
\text { diameter } \\
\text { (cm) }\end{array}$} & \multicolumn{4}{|c|}{ Day of sporulation } \\
\hline & TAM-D & TAM-N & TAM-S & TAM-T & & TAM-D & TAM-N & TAM-S & TAM-T \\
\hline A. niger PT-1 & $3.2 \pm 0.1$ & $2.9 \pm 0.1$ & $3.5 \pm 0.1$ & $1.0 \pm 0.1$ & $10.6 \pm 0.3$ & 4 & - & 5 & - \\
\hline A. sydowii PT-2 & $4.0 \pm 0.2$ & $3.3 \pm 0.1$ & $4.5 \pm 0.1$ & $1.8 \pm 0.1$ & $13.7 \pm 0.4$ & 5 & - & 4 & - \\
\hline A. pallidofulvus PT-3 & $2.2 \pm 0.2$ & $1.2 \pm 0.2$ & $2.6 \pm 0.1$ & 0 & $6.0 \pm 0.4$ & - & - & - & - \\
\hline A. sesamicola PT-4 & $2.2 \pm 0.1$ & $2.1 \pm 0.5$ & $2.4 \pm 0.2$ & $0.5 \pm 0.1$ & $7.2 \pm 0.2$ & 5 & - & 4 & - \\
\hline P. mangini PT-5 & $1.6 \pm 0.1$ & $1.5 \pm 0.2$ & $2.1 \pm 0.1$ & 0 & $5.2 \pm 0.4$ & - & - & - & - \\
\hline A. ustus PT-6 & $3.5 \pm 0.1$ & $2.7 \pm 0.1$ & $4.0 \pm 0.2$ & $1.8 \pm 0.1$ & $12.0 \pm 0.2$ & 4 & 5 & 4 & - \\
\hline A. tamarii PT-7 & $5.6 \pm 0.2$ & $4.6 \pm 0.2$ & $6.0 \pm 0.2$ & $3.0 \pm 0.3$ & $19.1 \pm 0.4$ & 3 & 4 & 2 & 4 \\
\hline
\end{tabular}

TAM-D theobromine agar media with dextrose as carbon source, TAM- $N$ theobromine agar media with ammonium sulphate as nitrogen source, TAM-S theobromine agar media with sucrose as carbon source, TAM-T theobromine agar media with theobromine as sole carbon and nitrogen source

in all given TLM. A. niger PT-1 just used the theobromine in TLM-S slightly. Only A. sydowii PT-2, A. ustus PT-6 and A. tamarii PT-7 could utilize theobromine largely in the liquid culture. The additional carbon source promoted theobromine utilization capacity of $A$. sydowii PT-2 and A. tamarii PT-7 through enhancement of cell density in the liquid culture [19]. Particularly, the highest theobromine removal rate was found in TLM-S for the potential theobromine-degrading fungi, including A. niger PT-1, A. sydowii PT-2, A. ustus PT-6 and A. tamarii PT-7. The composition of TLM-S was therefore chosen as the optimal medium to investigate theobromine degradation metabolites in the liquid culture.

\section{Theobromine degradation characterization in liquid culture}

A.niger PT-1, A. sydowii PT-2, A. ustus PT-6 and A. tamarii PT-7 were inoculated into TLM-S with an increasing theobromine concentration (100, 200 and 300 $\mathrm{mg} / \mathrm{L}$, respectively), and Tissue-culture bottles were incubated in an orbital shaker $\left(130 \mathrm{rpm}, 30^{\circ} \mathrm{C}\right)$, respectively. The inoculated bottles were took every $24 \mathrm{~h}$ for the determination of theobromine and related metabolites by using high-performance liquid chromatography (HPLC). Theobromine concentrations (Additional file 1: Table S1) are presented in Fig. 2. Significant difference $(p<0.05)$ was found in theobromine concentrations
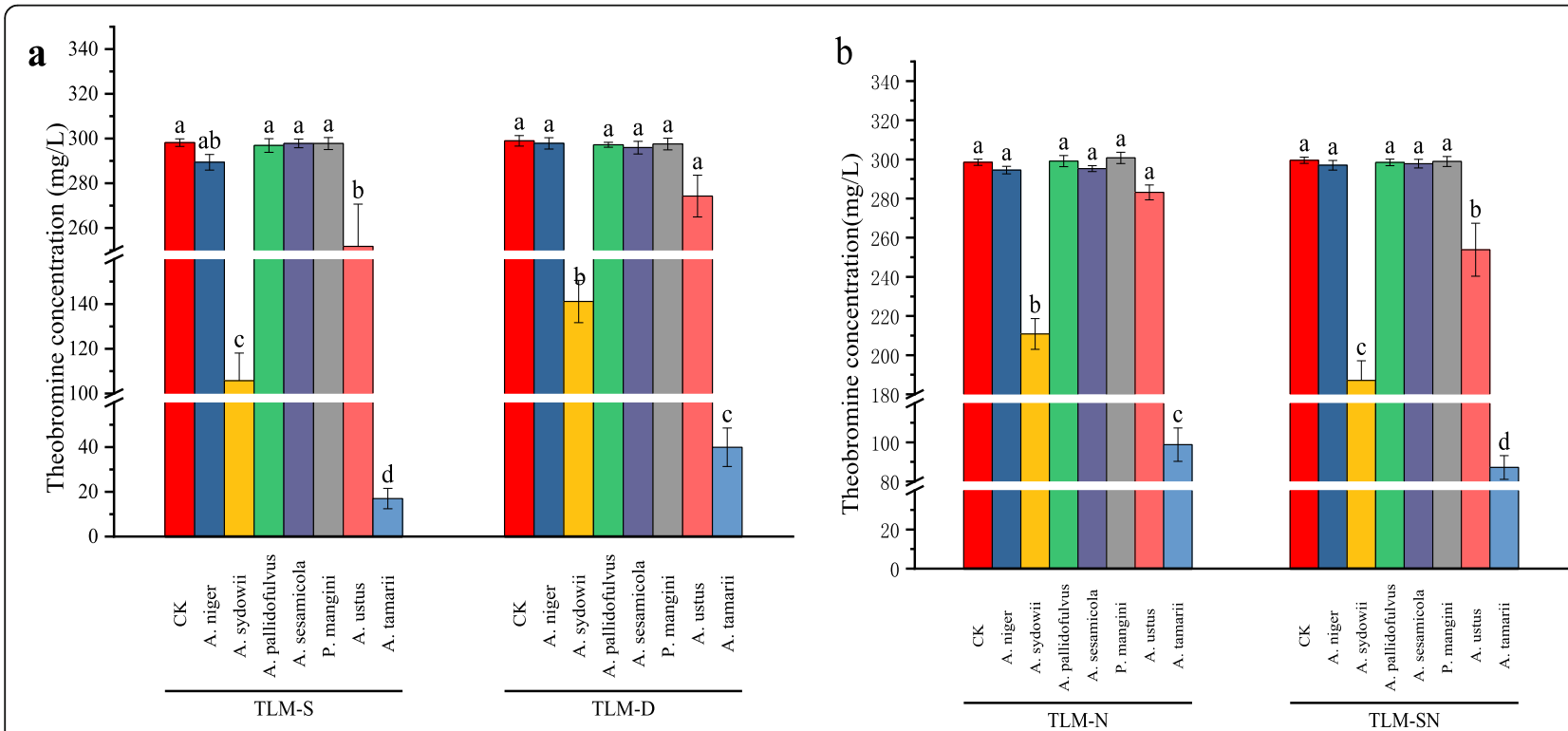

Fig. 1 Theobromine degradation capacity of tea-derived fungi in the liquid culture. a TLM-S = Theobromine liquid medium with sucrose as carbon source; TLM-D = Theobromine liquid medium with dextrose with sucrose as carbon source; $\mathbf{b}$ TLM-N = Theobromine liquid medium with ammonium sulphate as nitrogen source; TLM-SN = Theobromine liquid medium with sucrose and ammonium sulphate as carbon and nitrogen sources, respectively. Biocidal treatment without inoculation was defined as the control. All data were present by mean value \pm SD of three biological replications. The lowercase letters indicated a significant difference at $p<0.05$ levels by using Tukey's multiple comparison test for oneway ANOVA. The different letters show significant differences 


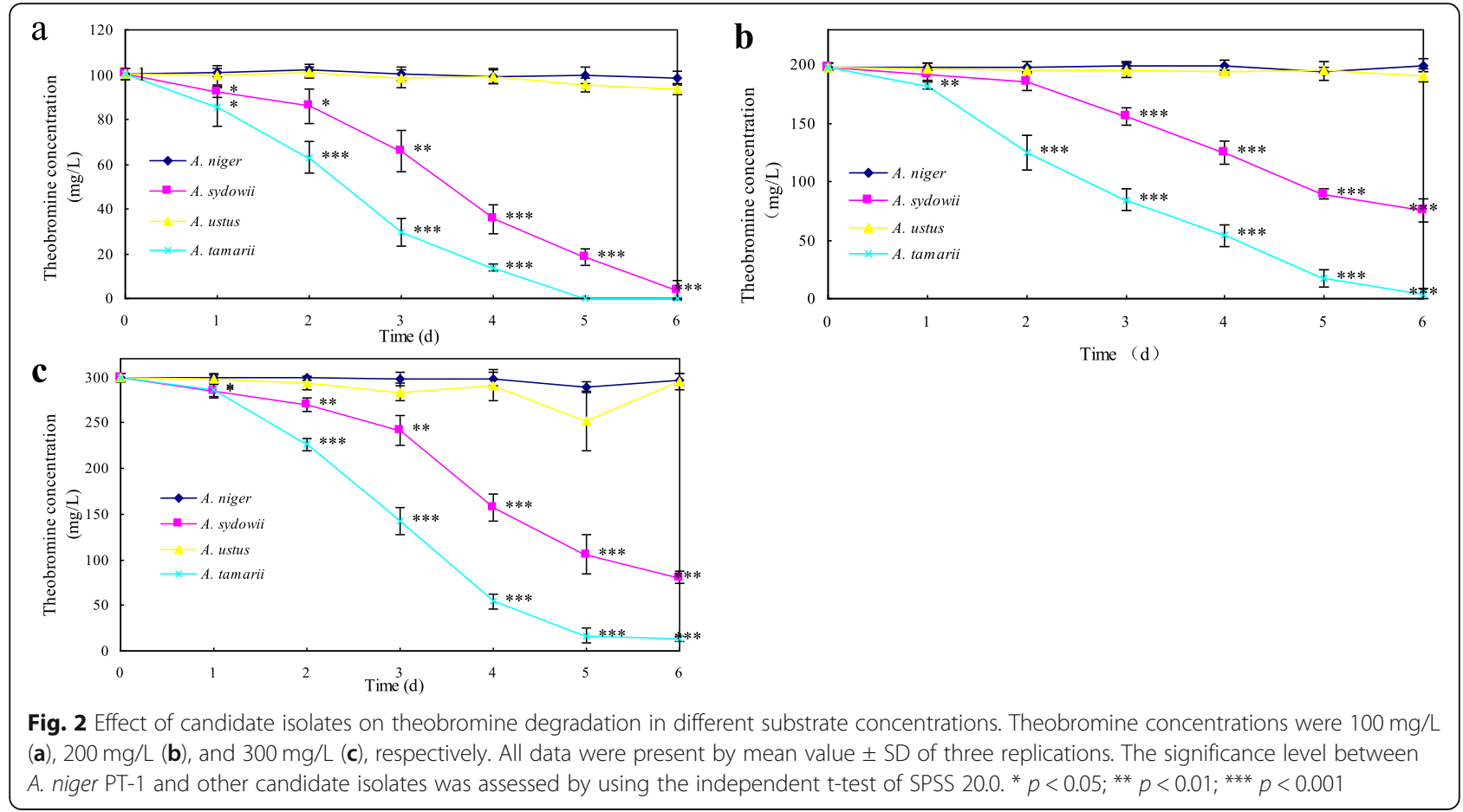

between four candidate isolates. Theobromine decreased slightly $(p>0.05)$ in all concentrations inoculated by $A$. niger PT-1 and A. ustus PT-6, which showed a limited theobromine utilization capacity in the liquid culture. In time-course experiments over a period of 6 days, $A$. tamarii PT-7 could degrade almost all the theobromine in the liquid culture. However, theobromine degradation capacity of A. sydowii PT-2 was limited with theobromine removal rates about 61.92 and $73.12 \%$ in high substrate concentrations of $200 \mathrm{mg} / \mathrm{L}$ and $300 \mathrm{mg} / \mathrm{L}$, respectively.

Theobromine catabolic intermediates were identified by HPLC using internal standard method (Table 2). 3,7Dimethyluric acid, 3-methylxanthine, 7-methylxanthine, 3-methyluric acid, xanthine and uric acid were detected consecutively in the liquid culture. The detected metabolites showed that both $\mathrm{N}$-demethylation and oxidation were found in theobromine catabolism. Quantitative analysis indicated that 3-methylxanthine was common and main demethylated metabolite through $\mathrm{N}$ demethylation at the N-7 position of theobromine in $A$. sydowii PT-2 and A. tamarii PT-7 culture. 7Methylxanthine was inferred as the demethylated product through the N-3 demethylation in A. tamarii PT-7 culture. Xanthine was a further demethylated metabolite found in A. tamarii PT-7 culture through N-3 demethylation of 3-methylxanthine or N-7 demethylation of 7methylxanthine. In A. ustus PT-6 and A. tamarii PT-7 culture, 3,7-dimethyluric acid, 3-methyluric acid and uric acid were direct oxidation products from theobromine, 3-methylxanthine and xanthine, respectively.

\section{Production of 3-methylxanthine through theobromine biodegradation}

3-Methylxanthine and other methylxanthines have been shown various biomedical effects as adenosine receptors and inhibitors of Primary Amine Oxidase [39, 40]. Due to high accumulation of 3-methylxanthine, 3-methylxanthine concentrations were determined by HPLC in A. sydowii PT-2 and $A$. tamarii PT-7 culture, respectively. 3-Methylxanthine concentrations are recorded in Additional file 1: Table S2

Table 2 Theobromine degradation metabolites detected in the liquid culture of four candidate isolates

\begin{tabular}{ll}
\hline Candidate isolates & Metabolites \\
\hline A. niger PT-1 & Not found \\
A. sydowii PT-2 & 3-Methylxanthine and xanthine \\
A. ustus PT-6 & 3,7-Dimethyluric acid and 3-methyluric acid \\
A. tamarii PT-7 & 3,7-Dimethyluric acid, 3-methylxanthine, 7-methylxanthine, 3-methyluric acid, xanthine and uric acid
\end{tabular}

TLM-S inoculated by candidate isolates were analyzed by HPLC for 3,7-dimethyluric acid, 3-methylxanthine, 7-methylxanthine, 3-methyluric acid, 7-methyluric acid, xanthine and uric acid 

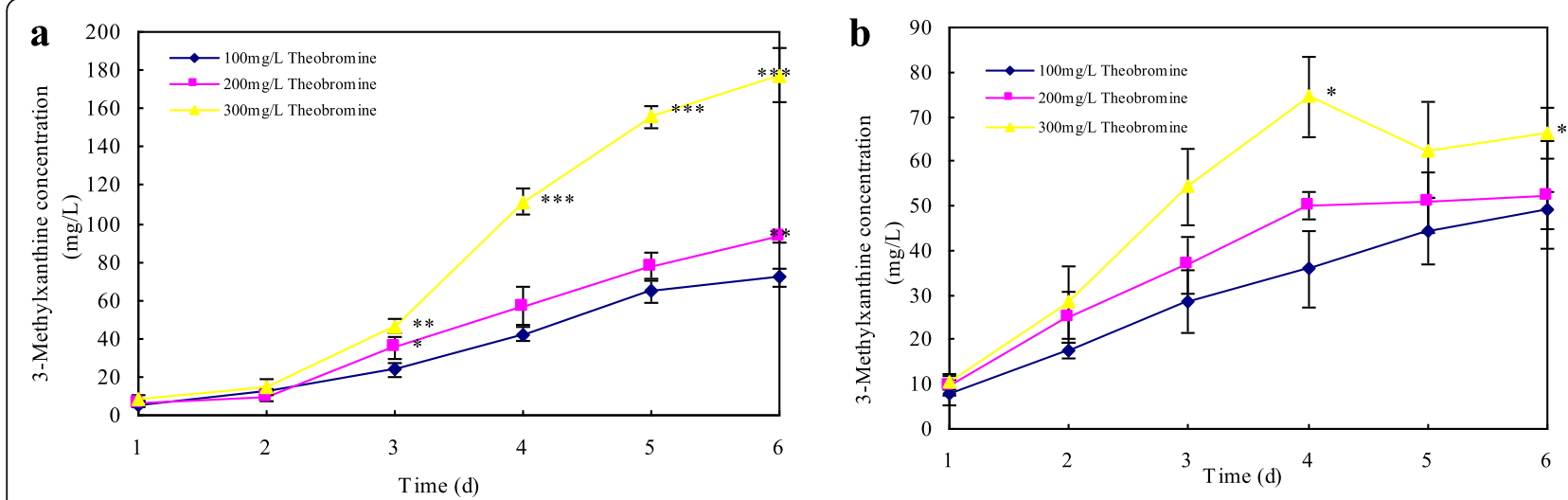

Fig. 3 Comparison of A. sydowii PT-2 (a) and A. tamarii PT-7 (b) on 3-menthylxanthine accumulation in the liquid culture. All data were present by mean value \pm SD of three biological replications. The significance level was assessed by using the independent t-test of SPSS 20.0 compared with that in $100 \mathrm{mg} / \mathrm{L}$ of theobromine. ${ }^{*} p<0.05 ;{ }^{* *} p<0.01 ;{ }^{* * *} p<0.001$

and presented in Fig. 3. The accumulation of 3methylxanthine increased along with theobromine degradation since it was detected in the liquid culture after cultivation for $24 \mathrm{~h}$. Over a 6 -day period cultivation of A. sydowii PT-2 (Fig. 3a), $71.84 \pm 4.44 \mathrm{mg} / \mathrm{L}, 92.81 \pm$ $2.86 \mathrm{mg} / \mathrm{L}$ and $177.12 \pm 14.06 \mathrm{mg} / \mathrm{L}$ of 3-methylxanthine were produced and increased significantly $(p<0.05)$ with an increasing initial theobromine concentration, respectively, showing a linear relationship between theobromine degradation and 3-methylxanthine accumulation. However, the accumulation of 3methylxanthine maintained at a low level about $66.31 \pm$ $5.68 \mathrm{mg} / \mathrm{L}$ in A. tamarii PT-7 culture with $300 \mathrm{mg} / \mathrm{L}$ theobromine, which was far below that in $A$. sydowii PT-2 culture. Generally, A. sydowii PT-2 showed its advantage in the production of 3-methylxanthine with $300 \mathrm{mg} / \mathrm{L}$ theobromine as feedstock.
The non-linear relationship between theorbromine degradation and 3-methylxanthine accumulation in $A$. tamarii PT-7 culture indicated that as the main intermediate of theobromine degradation, 3-methylxanthine might be degraded by A. tamarii PT-7 and other candidate isolates in the liquid culture. To investigate 3mehylxanthine metabolism, four candidate isolates were inoculated into a linearly increasing concentration of 3methylxanthine from $100 \mathrm{mg} / \mathrm{L}$ to $300 \mathrm{mg} / \mathrm{L}$, 3methylxanthine and related metabolites were determined by HPLC (Fig. 4). Compared with other isolates, A. sydowii PT-2 and A. tamarii PT-7 could reduce 3methylxanthine significantly $(p<0.05)$ in all given concentrations. Particularly, A. tamarii PT-7 degrade almost all 3-methylxanthine in a low substrate concentration (100 mg/L 3-methylxanthine), and maintained a relatively high removal rate about $34.97 \%$ in $300 \mathrm{mg} / \mathrm{L}$

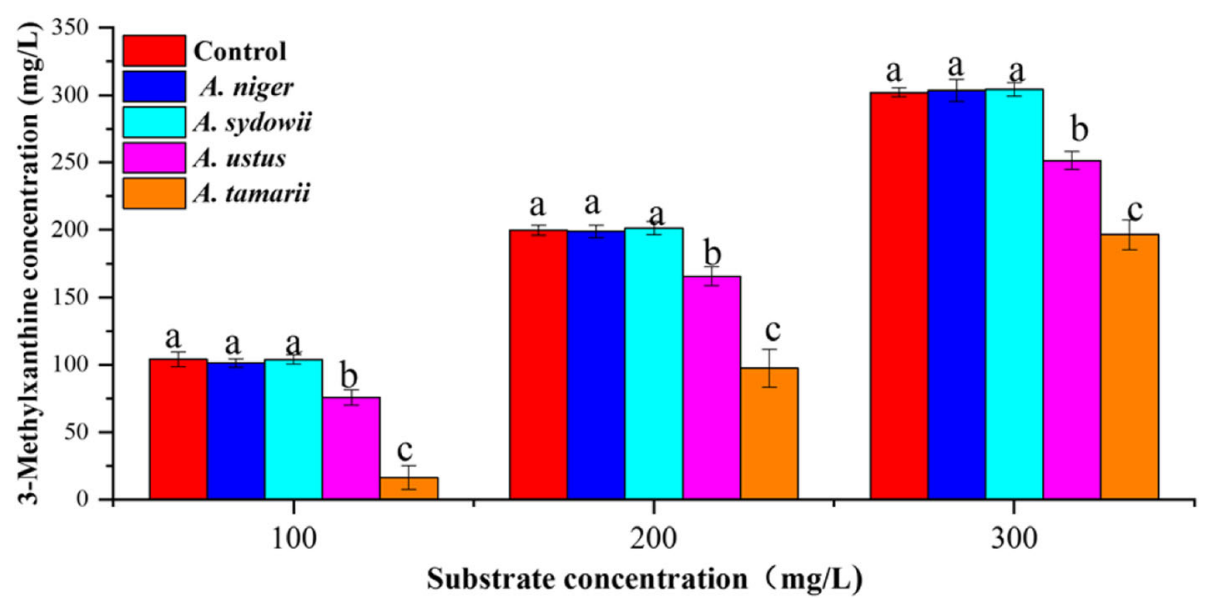

Fig. 4 Effect of candidate isolates on 3-methylxanthine metabolism. Biocidal treatment without inoculation was defined as the control. All data were present by mean value \pm SD of three biological replications. The lowercase letters indicated a significant difference at $p<0.05$ level by using one-way ANOVA of SPSS 20.0 between different candidate isolates at same substrate concentration. The different letters show significant differences 
substrate concentration after cultivation for 5 days. Through the analysis of related metabolites with 3methylxanthine degradation (Additional file 1: Table S3), 3-methyluric acid, xanthine and uric acid were detected in the liquid culture, respectively. Associated with the metabolites detected in theobromine degradation, xanthine was demethylated product from 3methylxanthine through N-3 demethylation. Alternatively, 3-methyluric acid and uric acid were direct oxidative products from 3-methylxanthine and xanthine at the C-8 position, respectively.

\section{Effects of $\mathrm{pH}$ and metal ions on 3-methylxanthine production}

The lower degradation capacity of 3-methylxanthine in liquid culture (Fig. 4) confirmed that A. sydowii PT-2 had application potential in production of 3methylxanthine with theobromine as feedstock. Metal ions and $\mathrm{pH}$ were principal factors influencing theobromine biodegradation and 3-methylxanthine production. Two series of experiments, such as a $\mathrm{pH}$ range from 3 to 7 and various metal ions, including $\mathrm{Fe}^{2+}, \mathrm{Ca}^{2+}, \mathrm{Mg}^{2+}$, $\mathrm{Mn}^{2+}, \mathrm{Cu}^{2+}$ and $\mathrm{Zn}^{2+}$, were prepared in TLM-S to investigate the influences of $\mathrm{pH}$ and metal ions, respectively. A. sydowii $\mathrm{PT}-2$ exhibited a high sensitivity to $\mathrm{pH}$, showing the best theobromine degradation and 3methylxanthine production at $\mathrm{pH} 5$ (Fig. $5 \mathrm{a}$ ). $\mathrm{Cu}^{2+}$ and $\mathrm{Zn}^{2+}$ restrained theobromine degradation and 3methylxanthine production significantly $(p<0.05)$, only $\mathrm{Fe}^{2+}$ promoted 3-methylxanthine production significantly $(p<0.05)$ compared with the control (Fig. 5b).

\section{Discussion}

Besides the traditional Traube synthesis, bioconversion offers an alternative way to produce 3-methylxanthine by using appropriate starter strain and precursor substances. Algharrawi et al. reconstructed an engineered Escherichia coli with genes $n d m A$ and $n d m D$ from $P$. putida, capable of producing 3-methylxanthine from exogenously fed theophylline [41]. Mckeague et al. engineered the eukaryotic microbial host Saccharomyces cerevisiae for the de novo biosynthesis of methylxanthines [42]. Additionally, 3-methylxanthine was main intermediate metabolite of theobromine through the $\mathrm{N}$ 7 demethylation by relevant fungi [31].

Because of dominant microbe in the solid-state fermentation causing the significant reduction of caffeine content [18], Pu-erh tea could be used to select effective strains converting theobromine to 3-methylxanthine. For seven tea-derived isolates, including $A$. niger PT-1, $A$. sydowii PT-2, A. pallidofulvus PT-3, A. sesamicola PT-4, P. manginii PT-5, A. ustus PT-6 and A. tamarii PT-7, five confirmed theobromine utilization capacity when cultured on TAM. The further screening in TLM showed that A. niger PT-1, A. sydowii PT-2, A. ustus PT-6 and A. tamarii PT-7 had relatively high theobromine degradation capacity in TLM-S after cultivation for 5 days at $30^{\circ} \mathrm{C}$. Particularly, A. sydowii PT-2 and A. tamarii PT-7 (Fig. 1) could degrade amount of theobromine (64.5 and $94.3 \%$, respectively).

It was established that the isolates generally preferred TLM-S in which extra sucrose enhanced theobromine degradation efficiency. Theobromine degradation efficiency of four candidate isolates was entirely different
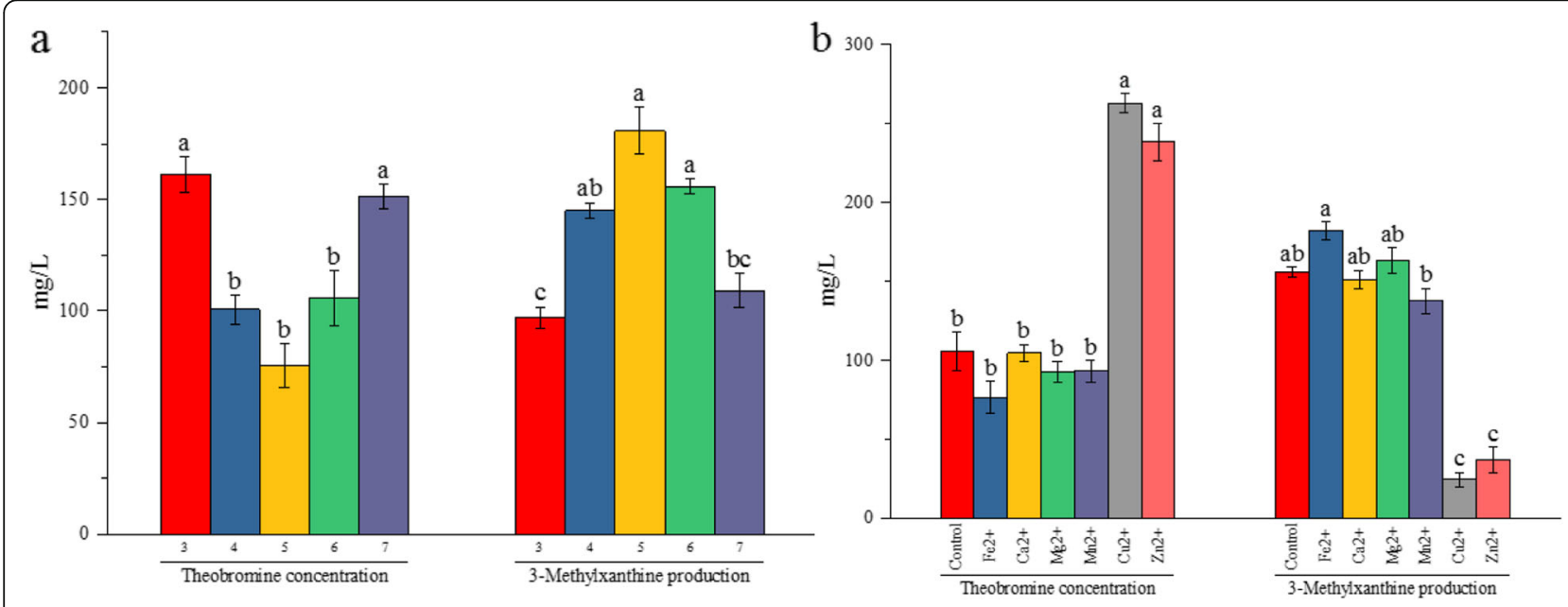

Fig. 5 Effects of $\mathrm{pH}$ (a) and metal ions (b) on theobromine degradation and 3-methylxanthine production in A. sydowii PT-2 culture, respectively. The $\mathrm{pH}$ in the inoculated mediums was adjusted by phosphate buffer. Metal ions were added into the culture solution at a concentration of 2 $\mathrm{mM}$. The culture solution without extra addition was defined as the control. The reaction $(\mathrm{pH} 6.0)$ was carried out at $30^{\circ} \mathrm{C}$ for 5 days on an incubator shaker (130 rpm). Data are presented as mean value \pm SD of three biological replications. The lowercase letters indicated a significant difference at $p<0.05$ level by using Tukey's multiple comparison test for one-way ANOVA. The different letters show significant differences 
(Fig. 2). Theobromine degradation capacity of $A$. niger PT-1 and A. ustus PT-6 were limited in the liquid culture, which might be related to cultivation method and medium components. For relatively high theobromine degradation efficiency, A. tamarii PT-7 and A. sydowii PT-2 were selected to investigate theobromine degradation pathway and application potential in the production of 3-methylxanthine.

The detected metabolites, including 3,7-dimethyluric acid, 3-methylxanthine, 7-methylxanthine, 3-methyluric acid, xanthine and uric acid, confirmed the existence of $\mathrm{N}$ demethylation and oxidation in theobromine catabolism. Ndemethylation happened at N-3 and N-7 positions in purine ring catalyzed by related $\mathrm{N}$-demethylase, and oxidation happened at the C-8 position catalyzed by related xanthine oxidase $[26,31,33]$. For $A$. sydowii PT-2, the likely theobromine catabolic process was described as follows: theobromine $\rightarrow 3-$ methylxanthine $\rightarrow$ xanthine. Except the above-mentioned process, other alternative processes also could be found in $A$. tamarii PT-7 culture. Such as the possible N-demethylation pathways were described as follows: theobromine $\rightarrow 7$-methylxanthine; 3,7-dimethyluric acid $\rightarrow 3$-methyluric acid. The possible oxidizing reaction happened in theobromine, 3methylxanthine and xanthine as precursor substance as follows: theobromine $\rightarrow$ 3,7-dimethyluric acid; 3methylxanthine $\rightarrow 3$-methyluric acid and xanthine $\rightarrow$ uric acid. We speculated that the related $\mathrm{N}$-demethylase and xanthine oxidase released by $A$. sydowii $\mathrm{PT}-2$ and $A$. tamarii PT-7 caused the difference in theobromine catabolism. Although caffeine catabolic pathways in tea plant and microorganisms are relatively clear [3, 4, 25-27], theobromine catabolic pathways in the liquid culture of potential microorganisms have not been defined, which deserves further research.

We confirmed that $A$. sydowii mainly produced theophylline through $\mathrm{N}$-demethylation at the N-7 position of caffeine, other $\mathrm{N}$-demethylated metabolites, such as 1,7-dimethylxanthine, 7-methylxanthine and 3-methylxanthine, were detected during tea fermentation, which showed that $A$. sydowii could release related $\mathrm{N}$-demethylase [43]. In this study, apart from caffeine, A. sydowii PT-2 also could remove the N-7 methyl of theobromine to formulate 3methylxanthine. Although $A$. ustus largely converted theophylline into 3-methylxanthine through the N-1 demethylation [19], absence of $\mathrm{N}$-demethylase removing the N-7 methyl limited theobromine degradation efficiency in the liquid culture. A. tamarii PT-7 exhibited broad-spectrum capacity in methylxanthines degradation, including theobromine, theophylline and 3-methylxanthine by releasing various $\mathrm{N}$-demethylases and oxidases, respectively. The high degradation of 3-methylxanthine reduced the accumulation of 3-methylxanthine in A. tamarii PT-7 culture. Therefore, A. sydowii PT-2 was best in production of 3methylxanthine with theobromine as feedstock.
Substrate concentration, $\mathrm{pH}$ and metal ions had profound impacts on theobromine degradation and 3methylxanthine production. A. sydowii PT-2 produced the maximum accumulation of 3-methylxanthine in the liquid culture of $300 \mathrm{mg} / \mathrm{L}$ theobromine. Comparisons showed that the optimal $\mathrm{pH}$ was 5 and $\mathrm{Fe}^{2+}$ promoted the conversion of theobromine into 3-methylxanthine significantly $(p<0.05)$, which provided optimum condition for the growth of $A$. sydowii PT-2 and enzymatic reaction of relevant $\mathrm{N}$-demethylase.

\section{Conclusions}

This paper describes related metabolites with theobromine degradation and explores potential application of tea-derived fungi in the production of 3-methylxanthine. Among seven microbial isolates from $\mathrm{Pu}$-erh tea, both $A$. sydowii PT-2 and A. tamarii PT-7 showed higher theobromine degradation capacity in TAM and TLM. 3,7-Dimethyluric acid, 3methylxanthine, 7-methylxanthine, 3-methyluric acid, xanthine and uric acid were detected by using HPLC in $A$. sydowii PT-2 and A. tamarii PT-7 culture, respectively, which confirmed the existence of $\mathrm{N}$-demethylation and oxidation in theobromine catabolism. Compared with that in A. tamarii PT-7 culture, 3-methylxanthine was accumulated largely in $A$. sydowii PT-2 culture along with theobromine degradation and showed a linear relation with initial theobromine concentration. A. sydowii PT-2 was an appropriate starter strain most suitable for the production of 3-methylxanthine, which could produce $177.12 \pm 14.06 \mathrm{mg} / \mathrm{L}$ 3-methylxanthine in TLM-S with $300 \mathrm{mg} / \mathrm{L}$ theobromine. Additionally, $\mathrm{pH}$ at 5 and metal ion of $\mathrm{Fe}^{2+}$ promoted the production of 3-methylxanthine significantly $(p<0.05)$. This paper presents an alternative way for 3-methylxanthine production through the microbial conversion of A. sydowii PT-2 with theobromine as feedstock.

\section{Methods}

\section{Strains and reagents}

Tea-derived fungal strains (Table 3) used in this study were isolated from Pu-erh tea and identified based on colony characteristics, conidial structure and PCR amplified sequences, and stored at $-20^{\circ} \mathrm{C}$ in our microbiology laboratory before further processing. Theobromine, 3,7dimethyluric acid, 3-methylxanthine, 7-methylxanthine, 3-methyluric acid, 7-methyluric acid, xanthine and uric acid were purchased from Sigma-Aldrich Co., Ltd. HPLC-grade acetonitrile and ammonium formate were purchased from Thermo Fisher Scientific Co., Ltd. Other reagents, including agar, dextrose, sucrose and ammonium sulphate, were analytical grade.

\section{Evaluation of growth of tea-derived fungi on theobromine agar medias}

For each strain, spore suspension was adjusted to $1.0 \times$ $10^{7} \mathrm{CFU} / \mathrm{mL}$ for inoculation after cultivation on PDA 
Table 3 Strains information of tea-derived fungi used in this work

\begin{tabular}{|c|c|c|c|c|c|c|}
\hline Isolate $^{a}$ & Primers & Fragments (bp) & Accession No. ${ }^{b}$ & Species & Strain No. & Homology \\
\hline PT-1 & ITS1/ITS4 & 546 & MT065763 & Aspergillus niger & NCBT 110A & $99.8 \%$ \\
\hline PT-2 & ITS1/ITS4 & 516 & MT065764 & Aspergillus sydowii & NRRL 250 & $99.8 \%$ \\
\hline \multirow[t]{3}{*}{ PT-3 } & ITS1/ITS4 & 541 & MT065765 & Aspergillus pallidofulvus & NRRL 4789 & $99.9 \%$ \\
\hline & $\mathrm{Bt} 2 \mathrm{a} / \mathrm{Bt} 2 \mathrm{~b}$ & 516 & MT084116 & & & \\
\hline & CF1L/CF4 & 765 & MT084120 & & & \\
\hline \multirow[t]{3}{*}{ PT-4 } & ITS1/ITS4 & 532 & MT065766 & Aspergillus sesamicola & CBS 137324 & $99.8 \%$ \\
\hline & $\mathrm{Bt} 2 \mathrm{a} / \mathrm{Bt} 2 \mathrm{~b}$ & 515 & MT084117 & & & \\
\hline & CF1L/CF4 & 757 & MT084121 & & & \\
\hline \multirow[t]{2}{*}{ PT-5 } & ITS1/ITS4 & 525 & MT065767 & Penicillium manginii & CBS 253.31 & $99.6 \%$ \\
\hline & $\mathrm{Bt} 2 \mathrm{a} / \mathrm{Bt} 2 \mathrm{~b}$ & 420 & MT084118 & & & \\
\hline \multirow[t]{2}{*}{ PT-6 } & ITS1/ITS4 & 502 & MT065768 & Aspergillus ustus & NRRL 275 & $100 \%$ \\
\hline & CF1L/CF4 & 694 & MT084122 & & & \\
\hline \multirow[t]{3}{*}{ PT-7 } & ITS1/ITS4 & 532 & MT065769 & Aspergillus tamarii & NRRL 20818 & $99.9 \%$ \\
\hline & $\mathrm{Bt} 2 \mathrm{a} / \mathrm{Bt} 2 \mathrm{~b}$ & 476 & MT084119 & & & \\
\hline & CF1L/CF4 & 715 & MT084123 & & & \\
\hline
\end{tabular}

${ }^{a}$ Those strains were stored in the microbiology laboratory of Yunnan Agricultural University with the number from PT-1 to PT-7, which can be accessed for reproducibility if need

${ }^{b}$ GenBank/EMBL/DDBJ accession number

media at $30^{\circ} \mathrm{C}$ for $72 \mathrm{~h}$, respectively [19]. Four kinds of TAM contained $20 \mathrm{~g} / \mathrm{L}$ agar and $600 \mathrm{mg} / \mathrm{L}$ theobromine were carried out to evaluate theobromine utilization, which included theobromine agar media with $2.0 \mathrm{~g} / \mathrm{L}$ dextrose as carbon source (TAM-D), theobromine agar media with $1.01 \mathrm{~g} / \mathrm{L}$ ammonium sulphate as nitrogen source (TAM-N), theobromine agar media with $2.0 \mathrm{~g} / \mathrm{L}$ sucrose as carbon source (TAM-S) and theobromine agar media only with theobromine as sole carbon and nitrogen source (TAM-T), respectively. Plates of each TAM were inoculated with $10 \mathrm{uL}$ spore suspension and incubated at $30^{\circ} \mathrm{C}$. At $24-\mathrm{h}$ intervals for 5 days, colony diameters were measured [44]. The isolated strains were categorized based on their total colony diameters as follows: low theobromine utilization $=$ diameter $\leq 7.9 \mathrm{~cm}$; moderate theobromine utilization $=$ diameter $8.0-15.9$ $\mathrm{cm}$; high theobromine utilization $=$ diameter $\geq 16.0 \mathrm{~cm}$. Tea-derived fungi selected for further study were those that showed at least moderate theobromine utilization (diameter $\geq 8.0 \mathrm{~cm}$ ) on agar medias.

\section{Assessment of theobromine-degrading fungi in theobromine liquid mediums}

Theobromine liquid medium (TLM) was prepared by using $4.0 \mathrm{~g} / \mathrm{L} \mathrm{NaNO}{ }_{3}, 1.3 \mathrm{~g} / \mathrm{L} \mathrm{KH}_{2} \mathrm{PO}_{4}, 0.19 \mathrm{~g} / \mathrm{L} \mathrm{Na} \mathrm{Na}_{2} \mathrm{H}-$ $\mathrm{PO}_{4} \cdot 7 \mathrm{H}_{2} \mathrm{O}, 0.26 \mathrm{~g} / \mathrm{L} \mathrm{CaCl} 2 \cdot 2 \mathrm{H}_{2} \mathrm{O}, 0.15 \mathrm{~g} / \mathrm{L} \mathrm{MgSO}_{4}, 2.0 \mathrm{~g} /$ $\mathrm{L}$ sucrose and $300 \mathrm{mg} / \mathrm{L}$ theobromine in distilled water [45]. To investigate the influence of carbon and nitrogen source on theobromine degradation, the modifications used either $5 \mathrm{~g} / \mathrm{L}$ sucrose or $10 \mathrm{~g} / \mathrm{L}$ dextrose as carbon source in theobromine liquid medium with sucrose as carbon source (TLM-S) or theobromine liquid medium with dextrose as carbon source (TLM-D), and $1.01 \mathrm{~g} / \mathrm{L}$ ammonium sulphate as nitrogen source in theobromine liquid medium with ammonium sulphate as nitrogen source (TLM-N), and $5 \mathrm{~g} / \mathrm{L}$ sucrose and $1.01 \mathrm{~g} / \mathrm{L}$ ammonium sulphate in theobromine liquid medium with sucrose and ammonium sulphate as carbon and nitrogen sources (TLM-SN), respectively. The spore suspension was adjusted to $1.0 \times 10^{7} \mathrm{CFU} / \mathrm{mL}$ for inoculation after eluting by using sterile saline solution with identical theobromine concentration. Both spore suspension and TLM were adjusted for $\mathrm{pH} 6.0$ by phosphate buffer. For each isolate, control and experimental mediums $(25 \mathrm{~mL}$ each) were inoculated with spore suspension with $4 \%$ inoculum size $(\mathrm{v} / \mathrm{v})$ that $1 \mathrm{~mL}$ spore suspension was inoculated into each medium, and biocidal treatment was defined as the control. Theobromine concentration was determined after cultivation at $30^{\circ} \mathrm{C}$ for 5 days on an incubator shaker (130 rpm), respectively.

\section{Analysis of theobromine degradation metabolites in liquid culture}

Through comparisons (Fig. 1), TLM-S therefore was chosen as the optimal medium to analyze theobromine degradation in the liquid culture. A series of TLM-S with different initial theobromine concentrations (100, 200 and $300 \mathrm{mg} / \mathrm{L}$, respectively) were set up and a 6-day period cultivation of each selected isolate were carried out on an incubator shaker $\left(130 \mathrm{rpm}, 30^{\circ} \mathrm{C}\right)$. At intervals of up to $24 \mathrm{~h}$ for 6 days, an aliquot of each culture was filtered through a 0.45 um syringe filter. Theobromine 
concentration and related metabolites were determined by HPLC using Agilent C18 chromatogram column $(250 \mathrm{~mm} \times 4.6 \mathrm{~mm}, 5 \mu \mathrm{m})$ with solvent A $(100 \%$ acetonitrile) and solvent $\mathrm{B}$ ( $0.1 \%$ ammonium formate) as mobile phase [19, 31].

Standard calibration curves were prepared from solutions of theobromine, 3-methylxanthine, 7methylxanthine, xanthine, 3,7-dimethyluric acid, 3methyluric acid, 7-methyluric acid and uric acid. Internal standard method was used to aid in the identification of metabolites related to theobromine catabolism [19]. 3Methylxanthine was quantificationally analyzed as the main intermediate metabolite in the liquid culture of $A$. sydowii PT-2 and A. tamarii PT-7, respectively.

\section{Influence of potential isolates on 3-methylxanthine metabolism}

3-Methylxanthine liquid mediums were prepared as above described with $5 \mathrm{~g} / \mathrm{L}$ sucrose as carbon source and a linearly increasing concentration of 3-methylxanthine from $100 \mathrm{mg} / \mathrm{L}$ to $300 \mathrm{mg} / \mathrm{L}$ to explore the effect of four candidate isolates, including $A$. niger PT-1, A. sydowii PT-2, A. ustus PT-6 and A. tamarii PT-7, respectively. Each candidate isolate was inoculated with $4 \%$ inoculum size (v/v) and 3-methylxanthine concentration was determined by HPLC after cultivation at $30{ }^{\circ} \mathrm{C}$ for 5 days on an incubator shaker (130 rpm), respectively.

\section{Effects of $\mathrm{pH}$ and metal ions on theobromine degradation} and 3-methylxanthine production

Effect of $\mathrm{pH}$ on theobromine degradation and 3methylxanthine production was investigated in TLM-S with a $\mathrm{pH}$ range from 3 to 7 adjusted by phosphate buffer $[18,46]$. In order to study the effect of metal ions, $\mathrm{Fe}^{2+}, \mathrm{Ca}^{2+}, \mathrm{Mg}^{2+}, \mathrm{Mn}^{2+}, \mathrm{Cu}^{2+}$ and $\mathrm{Zn}^{2+}$ were added into the culture solution in the form of salts (viz. FeS$\mathrm{O}_{4} \cdot 7 \mathrm{H}_{2} \mathrm{O}, \quad \mathrm{CaCl}_{2} \cdot 2 \mathrm{H}_{2} \mathrm{O}, \quad \mathrm{MgSO}_{4}, \quad \mathrm{MnSO}_{4} \cdot \mathrm{H}_{2} \mathrm{O}$, $\mathrm{CuSO}_{4} \cdot 5 \mathrm{H}_{2} \mathrm{O}, \mathrm{ZnSO}_{4} \cdot 7 \mathrm{H}_{2} \mathrm{O}$ ) at a concentration of $2 \mathrm{mM}$ and the culture solution without extra metal ions was defined as the control [32]. Theobromine and 3methylxanthine concentrations were determined after cultivation at $30^{\circ} \mathrm{C}$ for 5 days on an incubator shaker (130 rpm), respectively.

\section{Statistical analysis}

Three biological replications were carried out to ensure validity and repeatability. All data are presented as mean value \pm standard deviation (SD). The independent $t$-test and Tukey's multiple comparison tests for one-way analysis of variance (ANOVA) were carried out by using SPSS 20.0 for Windows to determine significant difference level.

\section{Supplementary information}

Supplementary information accompanies this paper at https://doi.org/10. 1186/s12866-020-01951-z.

Additional file 1: Table S1. Comparison of theobromine

concentrations detected by HPLC in liquid culture of different candidate isolates. Table S2. Production of 3-methylxanthine in TLM-S with different substrate concentrations inoculated by A. sydowii PT-2 and A. tamarii PT-7, respectively. Table S3. Related metabolites with 3-methylxanthine degradation detected in the liquid culture of different candidate isolates.

\section{Abbreviations}

CYP450: Cytochrome P450; TAM-D: Theobromine agar media with dextrose as carbon source; TAM-N: Theobromine agar media with ammonium

sulphate as nitrogen source; TAM-S: Theobromine agar media with sucrose as carbon source; TAM-T: Theobromine agar media with theobromine as sole carbon and nitrogen source; TLM: Theobromine liquid medium; TLM$\mathrm{S}$ : Theobromine liquid medium with sucrose as carbon source; TLM-

D: Theobromine liquid medium with dextrose as carbon source; TLM$\mathrm{N}$ : Theobromine liquid medium with ammonium sulphate as nitrogen source; TLM-SN: Theobromine liquid medium with sucrose and ammonium sulphate as carbon and nitrogen sources; HPLC: High-performance liquid chromatography; PCR: Polymerase chain reaction; CFU: Colony forming units; PDA: Potato dextrose agar; SD: Standard deviation; SPSS: Statistical product and service solutions; ANOVA: Analysis of variance

\section{Acknowledgements}

Not applicable.

\section{Authors' contributions}

ZBX and MCQ designed the work and contributed to the writing of the manuscript. ZCQ, MBS and MCQ carried out the laboratory work and statistical analysis. LXH participated in the revision. XT provided assistance in data analysis and revision. All authors approved the final version.

\section{Funding}

This work was supported by National Natural Science Foundation of China

(31960617), Modern Agricultural Industry Technology System of China (CARS-

23) and Open Funding Project of Henan Key Laboratory of Tea Plant

Comprehensive Utilization in South Henan (HNKLTOF2017006). The funding

bodies had no role in the design of the study, in data collection, analysis or interpretation, or in writing the manuscript.

\section{Availability of data and materials}

The data that support the findings of this study are available from the corresponding author upon reasonable request.

Ethics approval and consent to participate

Not applicable

Consent for publication

Not applicable.

\section{Competing interests}

The authors declare that they have no competing interests.

\section{Author details}

${ }^{1}$ College of Longrun Pu-erh Tea, Yunnan Agricultural University, Kunming 650201, Yunnan, China. ${ }^{2}$ Henan Key Laboratory of Tea Comprehensive Utilization in South Henan, Xinyang Agriculture and Forestry University, Xinyang 464000, Henan, China. ${ }^{3}$ Kunming Dapu Tea Industry Co., Ltd, Kunming 650224, Yunnan, China. ${ }^{4}$ State Key Laboratory of Tea Plant Biology and Utilization, Anhui Agricultural University, Hefei 230036, Anhui, China.

Received: 25 March 2020 Accepted: 18 August 2020

Published online: 27 August 2020

\section{References}

1. Anaya A, Cruz-Ortega R, Waller G. Metabolism and ecology of purine alkaloids. Front Biosci. 2006;11:2354-70. 
2. Georgiev K, Radeva-Ilieva M, Stoeva S, Zhelev I. Isolation, analysis and in vitro assessment of CYP3A4 inhibition by methylxanthines extracted from pu-erh and bancha tea leaves. Sci Rep. 2019;9:13941.

3. Mohanpuria P, Kumar V, Yadav SK. Tea caffeine: metabolism, functions, and reduction strategies. Food Sci Biotechnol. 2010;19:275-87.

4. Wang W, Zhu BY, Wang P, Deng WW, Wu GH, Wu FH, et al. Enantiomeric trimethylallantoin monomers, dimers, and trimethyltriuret: evidence for an alternative catabolic pathway of caffeine in tea plant. Org Lett. 2019;21:5147-51.

5. Wang D, Xu KL, Zhang Y, Luo X, Xiao R, Hou Y, et al. Acute and subchronic oral toxicities of Pu-erh black tea extract in Sprague-Dawley rats. J Ethnopharmacol. 2011;134:156-64.

6. Zhu BY, Chen LB, Lu MQ, Zhang J, Han JY, Deng WW, et al. Caffeine content and related gene expression: novel insight into caffeine metabolism in camellia plants containing low, normal and high caffeine concentrations. J Agric Food Chem. 2019;67:3400-11.

7. Qin JH, Li N, Tu PF, Ma ZZ, Zhang L. Change in tea polyphenol and purine alkaloid composition during solid-state fungal fermentation of postfermented tea. J Agric Food Chem. 2012;60:1213-7.

8. Li MY, Xiao Y, Zhong K, Bai JR, Wu YP, Zhang JQ, et al. Characteristics and chemical compositions of Pingwu Fuzhuan brick-tea, a distinctive post-fermentation tea in Sichuan province of China. Int J Food Prop. 2019;22(1):878-89.

9. Jiang HY, Shii T, Matsuo Y, Tanaka T, Jiang ZZ, Kouno I. A new catechin oxidation product and polymeric polyphenols of post-fermented tea. Food Chem. 2011;129:830-6.

10. Abe M, Takaoka N, Idemoto Y, Takagi C, Imai T, Nakasaki K. Characteristic fungi observed in the fermentation process for puer tea. Int J Food Microbiol. 2002;124:199-203.

11. Zhao M, Xiao W, Ma Y, Sun TT, Yuan WX, Na T, et al. Structure and dynamics of the bacterial communities in fermentation of the traditional Chinese post-fermented pu-erh tea revealed by 165 RNA gene clone library. World J Microbiol Biotechnol. 2013;29(10):1877-84.

12. Li ZY, Feng CX, Luo XG, Yao HL, Zhang DC, Zhang TC. Revealing the influence of microbiota on the quality of pu-erh tea during fermentation process by shotgun metagenomic and metabolomic analysis. Food Microbiol. 2018;76:405-15.

13. Zhao M, Su XQ, Nian B, Chen LJ, Zhang DL, Duan SM, et al. Integrated meta-omics approaches to understand the microbiome of spontaneous fermentation of traditional Chinese pu-erh tea. mSystems. 2019;4:e00680.

14. Zhang W, Yang RJ, Fang WJ, Yan L, Lu J, Sheng J, et al. Characterization of thermophilic fungal community associated with pile fermentation of pu-erh tea. Int J Food Microbiol. 2016;227:29-33.

15. Wang QP, Gong JS, Chisti Y, Sirisansaneeyakul S. Fungal isolates from a puerh type tea fermentation and their ability to convert tea polyphenols to theabrownins. J Food Sci. 2015;80(4):M809-17.

16. Zhao M, Zhang DL, Su XQ, Duan SM, Wan JQ, Yuan WX, et al. An integrated metagenomics/metaproteomics investigation of the microbial communities and enzymes in solid-state fermentation of pu-erh tea. Sci Rep. 2015;5:10117.

17. Zhang YJ, Skaar I, Sulyok M, Liu XZ, Rao MY, Taylor JW. The microbiome and metabolites in fermented pu-erh tea as revealed by high-throughput sequencing and quantitative multiplex metabolite analysis. PLoS One. 2016;11(6):e0157847.

18. Zhou BX, Ma CQ, Wang HZ, Xia T. Biodegradation of caffeine by whole cells of tea-derived fungi Aspergillues sydowii, Aspergillus niger and optimization for caffeine degradation. BMC Microbiol. 2018;18:53.

19. Zhou BX, Ma CQ, Xia T, Li XH, Zheng CQ, Wu T, et al. Isolation, characterization and application of theophylline-degrading Aspergillus fungi. Microb Cell Factories. 2020;19:72.

20. Zhou BX, Ma CQ, Ren XY, Xia T, Li XH, Wu Y. Production of theophylline via aerobic fermentation of pu-erh tea using tea-derived fungi. BMC Microbiol. 2019;19:261.

21. Arnaud MJ. Pharmacokinetics and metabolism of natural methylxanthines in animal and man. In: Fredholm BB, editor. Methylxanthines. Berlin: Springer Berlin Heidelberg; 2011. p. 33-91.

22. Hollingsworth RG, Armstrong JW, Campbell E. Pest control: caffeine as a repellent for slugs and snails. Nature. 2002;417:915-6.

23. Yu CL, Summers RM, Li Y, Mohanty SK, Subramanian M, Pope RM. Rapid identification and quantitative validation of a caffeine-degrading pathway in Psedomonas sp. CES. J Proteome Res. 2014;14:95-106.

24. Gummadi SN, Dash SS, Devarai S. Optimization of production of caffeine demethylase by Pseudomonas sp. in a bioreactor. J Int Microbiol Biotechnol. 2009;36:713-20.

25. Yu CL, Louie TM, Summers R, Kale Y, Gopishetty S, Subramanian M. Two distinct pathways for metabolism of theophylline and caffeine are coexpressed in Pseudomonas putida CBB5. J Bacteriol. 2009;191(14):4624-32.
26. Ma YX, Wu XH, Wu HS, Dong ZB, Ye JH, Zheng XQ, et al. Different catabolism pathway triggered by various methylxanthines in cafffeinetolerant bacterium Pseudomonas putida CT25 isolated from tea garden soil. J Microbiol Biotechnol. 2018;28(7):1147-55.

27. Nanjundaiah S, Mutturi S, Bhatt P. Modeling of caffeine degradation kinetics during cultivation of Fusarium solani using sucrose as co-substrate. Biochem Eng J. 2017;125:73-80.

28. Hakil M, Denis S, Gonzalez GV, Augur C. Degradation and product analysis of caffeine and related dimethyl xanthine by filamentous fungi. Enzym Microb Technol. 1998;22:355-9.

29. Brand D, Pandey A, Roussos S, Rsoccol C. Biological detoxification of coffee husk by filamentous fungi using a solid state fermentation system. Enzym Microb Technol. 2000;27(1):127-33.

30. Dash SS, Gummadi SN. Catabolic pathways and biotechnological applications of microbial caffeine degradation. Biotechnol Lett. 2006;28:1993-2002.

31. Mensah DO, Ocloo A, Lowor ST, Bonney EY, Okine LK, Adamafio A. Isolation and characterisation of theobromine-degrading filamentous fungi. Microbiol Res. 2018;206:16-24

32. Dash SS, Gummadi SN. Degradation kinetics of caffeine and related methylxanthines by induced cells of Pseudomonas sp. Curr Microbiol. 2007;55:56-60.

33. Mohapatra BR, Harris N, Nordin R, Mazumder A. Purification and characterization of a novel caffeine oxidase from Alcaligenes species. J Biotechnol. 2006;125:319-27.

34. Summers RM, Louie TM, Yu CL, Subramanian M. Characterization of a broad-specificity non-haem iron $\mathrm{N}$-demethylase from Pseudomonas putida CBB5 capable of utilizing several purine alkaloids as sole carbon and nitrogen source. Microbiology. 2011;157:583-92.

35. Summers RM, Seffernick JL, Quandt EM, Yu CL, Barrick JE, Subramanian MV. Caffeine junkie: an unprecedented glutathione S-transferase-dependent oxygenase required for caffeine degradation by Pseudomonas putida CBB5. J Bacteriol. 2013;195:3933-9.

36. Summers RM, Mohanty SK, Gopishetty S, Subramanian M. Genetic characterization of caffeine degradation by bacteria and its potential applications. Microb Biotechnol. 2015:8:369-78.

37. Mohanty SK, Yu CL, Das S, Louie TM, Gakhar L, Subramanian M. Delineation of the caffeine C-8 oxidation pathway in Pseudomonas sp. strain CBB1 via characterization of a new trimethyluric acid monooxygenase and genes involved in trimethyluric acid metabolism. J Bacteriol. 2012;194:3872-82.

38. Baggott MJ, Childs E, Hart AB, Bruin E, Palmer AA, et al. Psychopharmacology of theobromine in healthy volunteers. Psychopharmacology. 2013;228:109-18.

39. Shanahan P, O'Sullivan J, Tipton KF, Kinsella GK, Ryan BJ, Henehan GTM. Theobromine and related methylxanthines as inhibitors of primary amine oxidase. J Food Biochem. 2019;43:e12697.

40. Mitani T, Watanabe S, Yoshioka Y, Katayama S, Nakamura S, Ashida H. Theobromine suppresses adipogenesis through enhancement of CCAATenhancer-binding protein $\beta$ degradation by adenosine receptor A1. Biochim Biophys Acta, Mol Cell Res. 2017;1864:2438-48.

41. Algharrawi KH, Summers RM, Gopishetty S, Subramanian M. Direct conversion of theophylline to 3-methylxanthine by metabolically engineered E. coli. Microb Cell Factories. 2015;14:203-15.

42. Mckeague M, Wang $Y H$, Cravens A, Win MN, Smolke CD. Engineering a microbial platform for de novo biosynthesis of diverse methylxanthins. Metab Eng. 2016;38:191-203.

43. Zhou BX, Ma CQ, Ren XY, Xia T, Li XH. LC-MS/MS-based metabolomic analysis of caffeine-degrading fungus Aspergillus sydowii during tea fermentation. J Food Sci. 2020;85(2):477-85.

44. Ames JM, Guy RC, Kipping GJ. Effect of pH, temperature, and moisture on the formation of volatile compounds in glycine/glucose model systems. J Agric Food Chem. 2001;49(9):4315-23.

45. Gutierrez-Sanchez G, Atwood J, Kolli VSK, Roussos S, Augur C. Initial proteome analysis of caffeine-induced proteins in Aspergillus tamarii using two-dimensional fluorescence difference gel electrophoresis. Appl Biochem Biotechnol. 2012;166:2064-77.

46. Gokulakrishnan S, Chandraraj K, Gummadi SN. A preliminary study of caffeine degradation by Pseudomonas sp. GSC 1182. Int J Food Microbiol. 2007;113:346-50.

\section{Publisher's Note}

Springer Nature remains neutral with regard to jurisdictional claims in published maps and institutional affiliations. 